\title{
A New PLL Frequency Synthesis Structure
}

\author{
DAVID G. MESSERSCHMITT, MEMBER, IEEE
}

\begin{abstract}
In a PLL frequency synthesizer, the reference and VCO frequencies are divided down to their greatest common divisor frequency prior to phase comparison. We replace the dividers by a simplified form of digital frequency synthesizer, and examine the spectrum of the phase comparator outputs to show that the loop bandwidth can be dramatically increased, thereby improving the pull-in range and acquisition time.
\end{abstract}

\subsection{INTRODUCTION}

$\mathbf{T}$ WE purpose of a frequency synthesizer is to derive from a reference frequency $f_{1}$ a new frequency $f_{2}=K_{2} f_{1} / K_{1}$, where $K_{1}$ and $K_{2}$ are integers. One approach is to build a digital frequency synthesizer $[1,2]$, which requires that $K_{2}<$ $K_{1} / 2$ or $f_{1}>2 f_{2}$. The major advantage of this approach, which in some applications justifies its complexity, is that when the $K_{1}$ and $K_{2}$ are made programmable, the synthesized frequency can be changed virtually instantaneously. Another approach, which places no restrictions on $K_{1}$ and $K_{2}$, is the phase-locked loop (PLL) frequency synthesizer of Fig. 1(a) [2-5]. The reference frequency $f_{1}$ and voltage-controlled oscillator (VCO) frequency $f_{2}$ are divided down to their greatest common divisor frequency

$$
f_{0}=\frac{f_{1}}{K_{1}}=\frac{f_{2}}{K_{2}}
$$

for phase comparison.

Often the design of the loop filtering in Fig. 1 is a difficult task because there is a significant component at $f_{0}$ at the phase comparator output which must be highly attenuated to avoid significant $f_{0}$ sidebands on $f_{2}$. When $f_{0}$ is much smaller than $f_{2}$, this dictates a very narrow loop bandwidth, which results in a small pull-in range and slow acquisition [5]. One approach to increasing both pull-in range and acquisition speed is to add a frequency comparator to the phase comparator [6]. In this paper we will describe an alternative method of improving the loop acquisition properties. Specifically, we show that the loop bandwidth can be dramatically increased by modifying the divider circuits in Fig. 1.

The modified PLL synthesizer we will propose is in the same spirit as the digiphase synthesizer proposed by Gillette

Paper approved by the Editor for Data Communication Systems of the IEEE Communications Society for publication without oral presentation. Manuscript received November 24, 1977; revised February 16, 1978.

The author is with the Department of Electrical Engineering and Computer Sciences, University of California, Berkeley, CA 94720, on leave from Bell Laboratories, Holmdel, NJ 07733.

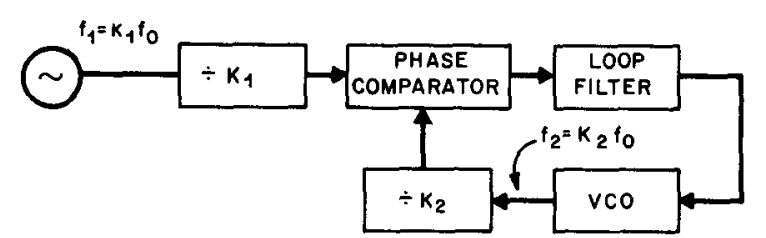

(a)

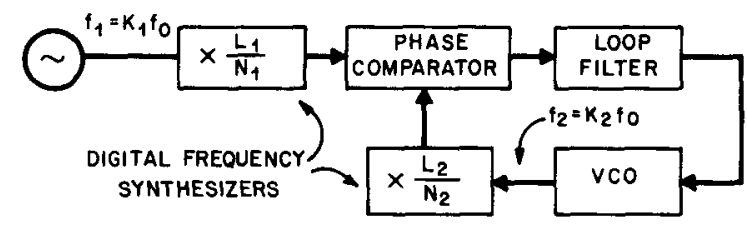

(b)

Fig. 1. PLL Frequency Synthesis. (a) PLL Synthesizer with $f_{0}$ Phase Comparison. (b) PLL Synthesizer Incorporating Digital Frequency Synthesizers.

[7]. However, our method has no restrictions on what frequency can be derived (the digiphase synthesizer requires that $K_{1}$ be of the form $2^{m} 5^{n}$ for base ten arithmetic or $2^{m}$ for base two arithmetic) and will generally require less hardware. Our work is also related to the concept of a "smooth pulse sequence" $[8,9]$ in that what we propose is essentially a new and simpler method of generating a smooth pulse sequence.

In Section 2.0 we describe our modified synthesizer. The bulk of the paper is then devoted to deriving the various spectra of interest in Section 3.0, with mathematical details relegated to the Appendices. This is a fairly challenging application of the principles of number theory [10]. A numerical example demonstrating the benefits of our approach is given in Section 4.0.

\subsection{MODIFIED PLL SYNTHESIZER}

We begin by noting that the dividers of Fig. 1(a) can be replaced by digital frequency synthesizers [1] as in Fig. 1(b). Thus, instead of simply dividing $f_{1}$ by $K_{1}$, we can multiply it by any rational fraction $L_{1} / N_{1}$, provided only that $2 L_{1}<N_{1}$. We can choose $L_{1}, N_{1}, L_{2}$, and $N_{2}$ such that both inputs to the phase comparator are at frequency $f_{p c}$, where

$$
f_{p c}=\frac{K_{1} L_{1}}{N_{1}} f_{0}=\frac{K_{2} L_{2}}{N_{2}} f_{0}
$$

Now $f_{\mathrm{pc}}$ can be chosen to be much larger than $f_{0}$, and since the fundamental frequency at the output of the phase comparator is $f_{p c}$, the loop bandwidth can be safely increased. 
The only advantage of Fig. 1(b) over a digital frequency synthesizer for deriving $f_{2}$ from $f_{1}$ directly is that there is no fundamental restriction on what $f_{2}$ can be derived in this manner. Relative to the PLL synthesizer of Fig. 1(a), Fig. 1(b) has much more hardware, and requires a more complicated analog phase comparator (since the digital synthesizers have analog sinusoid outputs).

We can simplify Fig. 1(b), and return to a digital phase comparator, by replacing the digital frequency synthesizer in Fig. 1(b) by a simpler circuit to be described in the next section. It will be shown in Section 3.0 that for the simpler circuit the phase comparator output fundamental returns to $f_{0}$, as in Fig. 1(a), but that the size of this fundamental and other low-frequency harmonics are dramatically reduced. Thus, the effect is much the same as if the loop filter had very narrow bandwidth, but without the impact on acquisition performance.

\subsection{Simple Approximate Digital Frequency Synthesizer}

Suppose we have available a clock at reference frequency $f$ and we wish to derive a new frequency $L f / N$. An exact digital frequency snythesizer $[1,2,11]$ generates a sequence of samples

$$
x_{L}(k)=A \sin (2 \pi L k / N)
$$

which repeat with period $N$,

$$
x_{L}(k+N)=x_{L}(k) \text {. }
$$

The implication of (2.3) is that only $N$ samples need to be stored to generate (2.2).* In particular, if we store the $N$ samples $x_{1}(k), 0 \leqslant k \leqslant N-1$, then (2.2) can be generated by the relation

$$
x_{L}(k)=x_{1}(k L \bmod N) .
$$

In (2.4) the function $n \bmod N$ is the remainder (an integer between 0 and $N-1$ ) when $n$ is divided by $N$.

In order to generate (2.4) by digital means we can store the samples $x_{1}(k)$ in a read-only memory (ROM), generate the address $M(k)=k L \bmod N$ digitally, and use a $\mathrm{D} / \mathrm{A}$ converter to generate the analog sample and a low-pass filter to reconstruct the analog sinusoid. This realization is shown in Fig. 2(a). We will take a closer look at the address generator in Section 2.2. The important points to note are that, for fixed $N$, a) the address can be represented by $n$ bits for the smallest $n$ such that $N \leqslant 2^{n}$ without fear of overflow since $k L \bmod N$ is always in the range 0 to $N-1$, and b) the frequency can be changed without altering the ROM contents simply by changing $L$ in the address generator as long as $N$ is fixed.

The D/A conversion introduces quantization noise to the synthesized frequency. This effect is analyzed in detail in [11], where it is shown that the output consists of harmonics of $f / N$, by far the largest being at $L f / N$, of course.

\footnotetext{
* These samples could also be recursively generated [1].
}

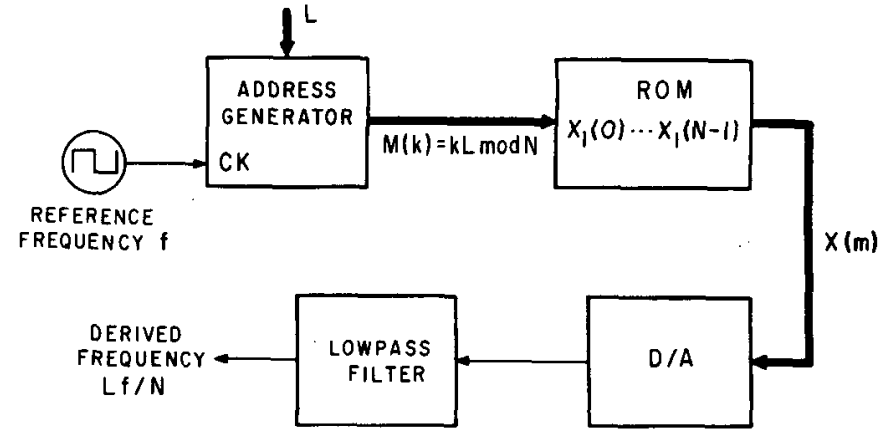

(a)

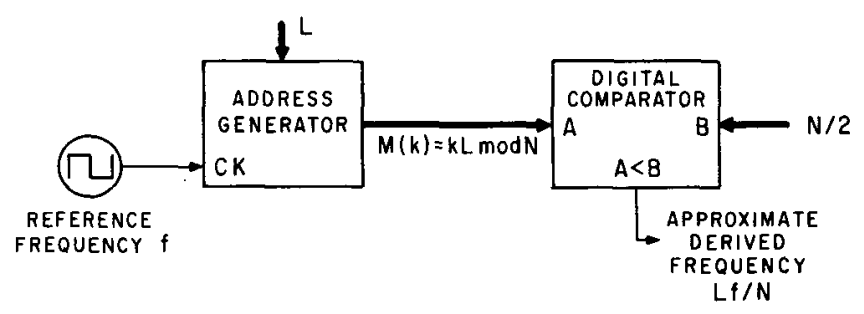

(b)

Fig. 2. Digital Frequency Synthesis. (a) A Digital Frequency Synthesizer. (b) A Simplified Approximate Synthesizer.

We can eliminate the $\mathrm{D} / \mathrm{A}$ converter entirely by representing $x_{1}(k)$ by a 1-bit quantization; that is, generate a one if $x_{1}(k)$ is positive or zero and generate a zero if $x_{1}(k)$ is negative. In fact, we can also then eliminate the ROM as well, since we see in (2.2) that

$$
x_{1}(k) \geqslant 0 \text { if } \begin{cases}0 \leqslant k \leqslant N / 2-1, & N \text { even } \\ 0 \leqslant k \leqslant \frac{N-1}{2}, & N \text { odd. }\end{cases}
$$

Therefore, the ROM can be replaced, as in Fig. 2(b), by a digital comparator which compares two $n$-bit binary numbers** (not to be confused with a phase comparator). Although this approximation is coarse, the PLL acts to suppress the relatively large sidebands. The relevant spectra are calculated in Section 3.0. Figure 2(b) is the approximate synthesizer which we propose as a replacement for the exact synthesizer in Fig. 1(b).

At this point the reader would probably benefit from a simple example. Let $N=8$, and suppose we desire to derive the frequency $3 f / 8$ where we have available a clock at rate $f$. Then we generate the sequence of addresses of Table 1 . The resulting approximate representation of $3 f / 8$ is shown in Fig. 3. This waveform has three transitions for every eight transitions of $f$, and repeats itself after every eight transitions of $f$. It has a fundamental frequency $f / 8$, but the largest harmonic is the third, at $3 f / 8$. The PLL can suppress the undesired harmonics to any extent desired.

One way to view the circuit of Fig. 2(b) is that it attempts to approximate frequency $L f / N$ with a waveform which has

\footnotetext{
** If $N$ is a power of two, this comparator is not needed. The approximate sinusoid is just the most significant bit of the address.
} 
TABLE 1

\begin{tabular}{cccc}
\hline$k$ & $M(k)=3 k \bmod 8$ & $M(k) \leqslant 3 ?$ & $\begin{array}{c}\text { Digital } \\
\text { Comparator } \\
\text { Output }\end{array}$ \\
\hline 0 & 0 & YES & 1 \\
1 & 3 & YES & 1 \\
2 & 6 & NO & 0 \\
3 & 1 & YES & 1 \\
4 & 4 & NO & 0 \\
5 & 7 & NO & 0 \\
6 & 2 & YES & 1 \\
7 & 5 & NO & 0 \\
& (PERIODIC REPETITION THEREAFTER)
\end{tabular}

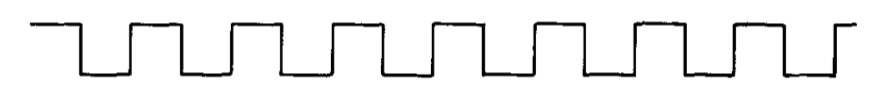

(a)

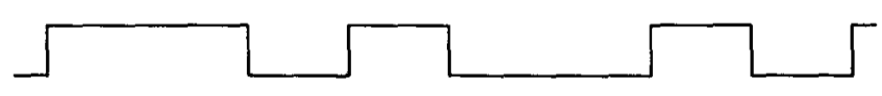

(b)

Fig. 3. Example Waveforms for $N=8, L=3$. (a) Reference Frequency $f$. (b) Derived Approximate Frequency $3 f / 8$.

transitions corresponding to transitions of reference frequency $f$. In particular, it chooses the transition of $f$ closest to the actual transition of exact frequency $L f / N$. The maximum error, or jitter, is thus one half period of $f$, or $1 / 2 f \mathrm{~s}$. As $f$ gets large relative to $L f / N$, the significance of this jitter diminishes. The PLL acts to reduce this jitter.

In some applications the approximate synthesized waveform may be adequate without further jitter reduction in a PLL. For example, as a clock for a digital logic circuit, the residual jitter may be small enough to be of no consequence.

\subsection{Address Generator}

The address generator of Fig. 2 is readily designed from the recursive relation

$$
\begin{aligned}
M(k+1)= & (k+1) L \bmod N \\
= & (k L \bmod N+L) \bmod N \\
& (M(k)+L) \bmod N .
\end{aligned}
$$

Writing this relationship in another way,

$$
M(k+1)= \begin{cases}M(k)+L, & 0 \leqslant M(\dot{k}) \leqslant N-1-L \\ M(k)+L-N, & N-L \leqslant M(k) \leqslant N-1\end{cases}
$$

we get the implementation of Fig. 4. The address $M(k)$ is stored in the clocked latch while the new address $M(k+1)$ is determined. The latter is generated by comparing $M(k)$ to $N-L$; if $M(k)<N-L$ then $L$ is added to $M(k)$, and otherwise $L-N$ (a negative number) is added. The added complexity of this circuit relative to the divide by $K_{1}$ and $K_{2}$ counters of

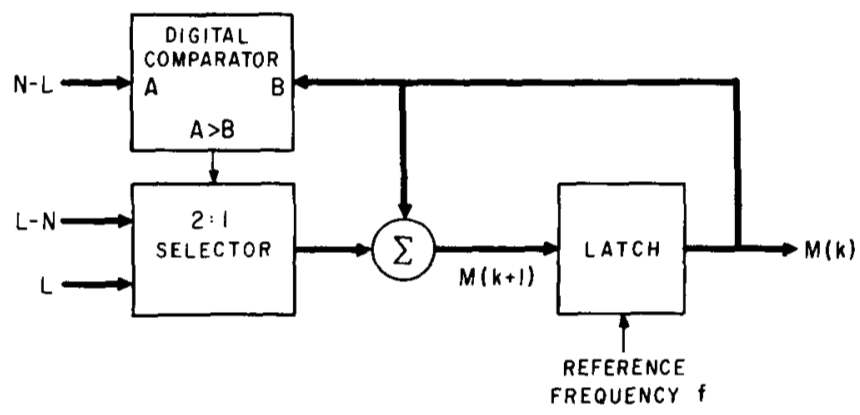

Fig. 4. Address Generator.

Fig. 1(a) will of course limit the usefulness of this approach to slightly lower frequencies.

\subsection{RELEVANT SPECTRA}

We will now calculate the spectra of the approximate synthesized frequency of Fig. 2(b) and the output of the phase comparator of Fig. 1(b) when the approximate digital frequency synthesizer of Fig. 2(b) replaces the two exact synthesizer boxes and an exclusive-or phase detector is used. The most important result is that the fundamental of the phase comparator output is $f_{0}$, just as in Fig. 1(a), as long as $N_{1}$ and $N_{2}$ contain no common factors other than unity (otherwise the fundamental is a subharmonic of $f_{0}$, a situation to be avoided). A numerical example in Section 4.0 will demonstrate, however, that this fundamental and the lower frequency harmonics have been dramatically reduced relative to Fig. 1(a).

We can use the model of Fig. 5 to analyze the in-lock performance of the PLL synthesizer. The clocks $f_{1}$ and $f_{2}$ we consider to be counted down from a fictitious high-speed clock with frequency $K_{1} K_{2} f_{0}$, the least common multiple of $f_{1}$ and $f_{2}$, in order to make the problem a simpler discrete one. This has the unimportant affect of restricting the relative phase of the two clocks to discrete multiples of $\left(K_{1} K_{2} f_{0}\right)^{-1} \mathrm{~s}$. The inputs and outputs of the exclusive-or phase comparator*** assume the values +1 and 0 , and obey the relation

$$
z_{k}=2 r_{k+k_{0}} s_{k}-r_{k+k_{0}}-s_{k}+1
$$

where the index $k$ runs at frequency $K_{1} K_{2} f_{0}$ throughout. The relative phase of the two inputs is determined by $k_{o}$.

\subsection{Periodicity of Phase Detector Output}

Using the notation $(m, n)$ to denote the greatest common divisor of $m$ and $n$, we henceforth assume that $\left(K_{1}, K_{2}\right)=$ $\left(L_{1}, N_{1}\right)=\left(L_{2}, N_{2}\right)=1$ (which we call relatively prime) since any common factors in these pairs of integers can be divided out without modifying any of the frequencies generated. We make no restriction on $\left(L_{1}, L_{2}\right)$ and $\left(N_{1}, N_{2}\right)$,

$$
\begin{aligned}
& \left(L_{1}, L_{2}\right)=d_{L} \\
& \left(N_{1}, N_{2}\right)=d_{N} .
\end{aligned}
$$

*** A set-reset flip-flop could also be used with similar results. 


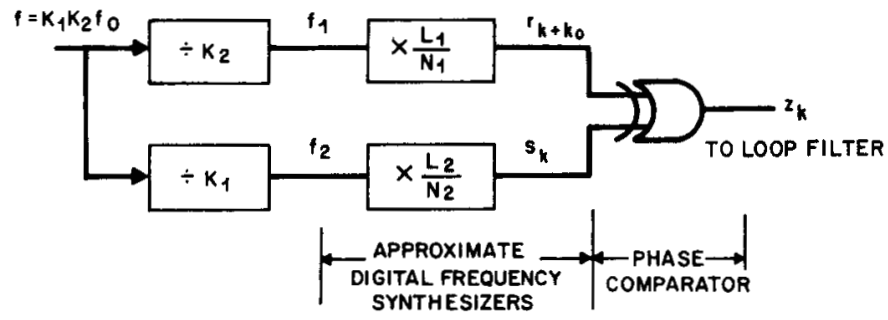

Fig. 5. Model of an In-Lock PLL.

It follows that $\left(d_{L}, d_{N}\right)=1$. From $(2.1)$ there is the constraint

$$
1=\frac{K_{1} L_{1} N_{2}}{K_{2} N_{1} L_{2}}=\frac{K_{1}}{K_{2}} \frac{\left[\frac{L_{1}}{d_{L}}\right]\left[\frac{N_{2}}{d_{N}}\right]}{\left[\frac{N_{1}}{d_{N}}\right]\left[\frac{L_{2}}{d_{L}}\right]}
$$

which through factor analysis can be shown to imply that

$$
\begin{aligned}
& K_{1}=\frac{N_{1} L_{2}}{d_{N} d_{L}} \\
& K_{2}=\frac{L_{1} N_{2}}{d_{N} d_{L}}
\end{aligned}
$$

It is evident that $r_{k}$ is periodic in $K_{2} N_{1}$, while $s_{k}$ is periodic in $K_{1} N_{2}$, and hence the minimum period $N$ of $z_{k}$ must satisfy

$$
N=\alpha K_{2} N_{1}=\beta K_{1} N_{2}
$$

for relatively prime integers $\alpha$ and $\beta$. Hence, from (3.4) and (3.5),

$$
\frac{\alpha}{\beta}=\frac{K_{1} N_{2}}{K_{2} N_{1}}=\frac{L_{2}}{L_{1}}
$$

and it follows that

$$
\begin{aligned}
& \alpha=L_{2} / d_{L} \\
& \beta=L_{1} / d_{L} \\
& N=K_{2} L_{2} N_{1} / d_{L}=K_{1} L_{1} N_{2} / d_{L} .
\end{aligned}
$$

The fundamental frequency of $z_{k}$ is therefore

$$
\frac{K_{1} K_{2} f_{0}}{N}=\frac{f_{0}}{d_{N}}
$$

so that there is a strong motivation to keep $d_{N}=1\left(N_{1}\right.$ and $N_{2}$ relatively prime).

\subsection{Spectra of Phase Detector Inputs}

The discrete Fourier transform (DFT) of the input sequence $r_{k}$ is

$$
R_{p}=\frac{1}{K_{2} N_{1}} \sum_{k=0}^{K_{2} N_{1}-1} r_{k} e^{-j 2 \pi k p / K_{2} N_{1}}
$$

A similar relationship holds for $S_{p}$, the DFT of $s_{k}$, with $K_{1} N_{2}$ substituted for $K_{2} N_{1}$. It is shown in Appendix A that for $p \neq 0$,

$$
R_{p}=\frac{\left[1-e^{-j 2 \pi p / N_{1}}\right]\left[1-(-1)^{p J_{1}} z\right]}{K_{2} N_{1}\left[1-e^{-j 2 \pi p / K_{2} N_{1}}\right]\left[1-e^{-j 2 \pi p J_{1} / N_{1}}\right]}
$$

where

$$
z= \begin{cases}1, & N_{1} \text { even } \\ e^{-j \pi p J_{1} / N_{1}}, & N_{1} \text { odd }\end{cases}
$$

and where $J_{1}$ is the unique integer in $\left[0, N_{1}-1\right]$ which satisfies

$$
J_{1} L_{1} \bmod N_{1}=1
$$

In addition, when $p=0$,

$$
R_{0}=\left\{\begin{array}{ll}
1 / 2, & N_{1} \text { even } \\
\left(N_{1}+1\right) / 2 N_{1}, & N_{1} \text { odd }
\end{array} .\right.
$$

\subsection{Spectrum of Phase Detector Output}

It is shown in Appendix B that the DFT of $z_{k}$ is

$$
\begin{aligned}
Z_{p}= & \frac{1}{N} \sum_{k=0}^{N-1} z_{k} e^{-j 2 \pi k p / N} \\
= & \delta_{p, 0}+\sum_{k=0}^{K-1} e^{j 2 \pi k_{0} p_{1} / K_{2} N_{1} R_{p_{1}} S_{p_{2}}} \\
& -e^{j 2 \pi k_{0} / N} R_{d_{L} p / L_{2}} M_{2}(p)-S_{d_{L} / L_{1}} M_{1}(p)
\end{aligned}
$$

where

$$
\begin{aligned}
& K=K_{2} N_{1} d_{L} / L_{1} \\
& p_{1}=p_{1} *+L_{1} k / d_{L} \\
& p_{2}=\left(p_{2} *-L_{2} k / d_{L}\right) \bmod K_{1} N_{2}
\end{aligned}
$$

and $0 \leqslant p_{1} *<L_{1} / d_{L}$ and $p_{2} *$ uniquely satisfies

$$
L_{2} p_{1} * / d_{L}=p \bmod L_{1} / d_{L}
$$

and

$$
p_{2} *=\frac{d_{L} p-L_{2} p_{1} *}{L_{1}}
$$


and the function $M_{k}(p)$ is unity if $d_{L} p / L_{k}$ is an integer and zero otherwise.

\subsection{NUMERICAL EXAMPLE}

So far we have not discussed how the integers $L_{1}, N_{1}, L_{2}$, $N_{2}$ should be chosen other than that they must satisfy (2.1) and that it is advantageous if $d_{N}=1$. Ideally they would be chosen to minimize the jitter on derived frequency $f_{2}$ for some desired loop filter. A simpler strategy, which works as long as one of $f_{1}$ or $f_{2}$ is more than twice as large as the other, is to choose $L_{1}=N_{1}=1$ if $f_{2}>2 f_{1}$ or $L_{2}=N_{2}=1$ if $f_{1}>2 f_{2}$. This has the advantage of eliminating one of the approximate digital frequency synthesizers.

As a numerical example, we consider the case $K_{1}=193$ and $K_{2}=2048$ (this example is motivated by two frequencies prevalent in the Bell System digital transmission and switching networks). It follows that $L_{2}=K_{1}$ and $N_{2}=K_{2}$ if we choose $L_{1}=N_{1}=1$ : Note that in this case the reference $f_{1}$ is the lower frequency, the fundamental frequency at the phase detector output is $f_{0}=f_{1} / 193$, and the nominal frequency at the phase detector input is $f_{p c}=f_{1}=193 f_{2} / 2048$.

At the output of the phase detector, the fundamental $f_{0}$ and lower harmonics were found to have little dependence on the phase $k_{o}$, so the higher harmonics were examined for $k_{o}=$ 512 using (3.13). The first very significant harmonic is $386 f_{0}$ (the second harmonic of $f_{1}$ ), for which $C_{386}=-10.5 \mathrm{~dB}$, where

$$
C_{k}=20 \log _{10}\left|Z_{k}\right| \text {. }
$$

For most $k<386, C_{k}$ is in the range of $-40 \mathrm{~dB}$ or greater, with odd harmonics including $f_{0}$ virtually nonexistent. The only exceptions are $C_{118}=-30 \mathrm{~dB}, C_{150}=-37 \mathrm{~dB}, C_{236}=$ $-36 \mathrm{~dB}$, and $C_{268}=-31 \mathrm{~dB}$. This performance is quite favorable when compared with that of Fig. 3(a), where $C_{1}=$ $-10 \mathrm{~dB}$. The addition of the approximate synthesizer therefore adds more than $30 \mathrm{~dB}$ additional suppression of all harmonics out to the 118th thereby greatly easing the loop filtering requirement.

\subsection{CONCLUSION}

The modification of the PLL synthesizer we have described would appear to deserve consideration in many applications. The increase in circuit complexity when two dividers are replaced by a single address generator and comparator is not great, and programmability of the synthesized frequency is just as simple. The benefits in terms of improved acquisition and/or reduced sidebands on the synthesized frequency have been demonstrated.

\section{APPENDIX A}

It is convenient to write the two-level sinusoid approximation as

$$
x_{1}(k)=\left\{\begin{array}{ll}
\psi_{[0, N / 2-1]^{(k)}}, & N \text { even } \\
\psi_{[0,(N-1) / 2]}^{(k)}, & N \text { odd }
\end{array}\right\}
$$

where the indicator function $\psi$ is defined as

$$
\psi_{\mathrm{s}}(k)=\left\{\begin{array}{ll}
1, & k \in S \\
0, & k \notin S
\end{array}\right\} .
$$

Equations for $r_{k}$ and $s_{k}$ can then be obtained as follows: Divisions of the $K_{1} K_{2} f_{0}$ clock by $K_{1}$ and $K_{2}$ are obtained by defining new indexes $m$ and $n$

$$
\begin{array}{ll}
k=m K_{2}+i, & 0 \leqslant i \leqslant K_{2}-1 \\
k=n K_{1}+j, & 0 \leqslant j \leqslant K_{1}-1
\end{array}
$$

from which, from (A.1) and (2.4),

$$
\begin{aligned}
& r_{k}=\psi_{\left[0, N_{1} / 2-1\right]}\left(m L_{1} \bmod N_{1}\right) \\
& s_{k}=\psi_{\left[0, N_{2} / 2-1\right]}\left(n L_{2} \bmod N_{2}\right)
\end{aligned}
$$

for $N$ even, with similar equations for $N$ odd.

Expression (3.9) will now be derived. Substituting (A.3) and (A.4) into (3.8)

$$
\begin{aligned}
R_{p}= & \frac{1}{K_{2} N_{1}} \sum_{i=0}^{K_{2}-1} \sum_{m=0}^{N_{1}-1} \psi_{\left[0, N_{1} / 2-1\right]}\left(m L_{1} \bmod N_{1}\right) \\
& \cdot e^{-j 2 \pi\left(m K_{2}+i\right) p / K_{2} N_{1}} \\
= & \frac{1}{K_{2} N_{1}} \frac{1-e^{-j 2 \pi p / N_{1}}}{1-e^{-j 2 \pi p / K_{2} N_{1}}} \sum_{m=0}^{N_{1}-1} \psi_{\left[0, N_{1} / 2-1\right]} \\
& \cdot\left(m L_{1} \bmod N_{1}\right) e^{-j 2 \pi m p / N_{1}} .
\end{aligned}
$$

The remaining sum can be evaluated by letting

$$
q=m L_{1} \bmod N_{1}
$$

which has inverse relationship [11]

$$
m=q J_{1} \bmod N_{1}
$$

where $J_{1}$ is given by (3.11). Then the sum in (A.5) becomes

$$
\sum_{q=0}^{N_{1}-1} \psi_{\left[0, N_{1} / 2-1\right]}(q) e^{-j 2 \pi q p J_{1} / N_{1}}
$$

and (3.9) follows immediately. A minor modification yields a similar formula for $N$ odd.

\section{APPENDIX B}

The DFT of (3.13) will now be evaluated for the $z_{k}$ of (3.1). Substituting (3.8) and a similar equation for $S_{p}$ into (3.1) and (3.13) 


$$
\begin{aligned}
Z_{p}= & \delta_{p, 0}+2 \sum_{p_{1}=0}^{K_{2} N_{1}-1} \sum_{p_{2}=0}^{K_{1} N_{2}-1} e^{j 2 \pi k_{0} p_{1} / K_{2} N_{1}} \\
& \cdot R_{p_{1}} S_{p_{2}} \psi_{I_{1}}\left(p_{1}, p_{2}\right)-e^{j 2 \pi k_{0} / N} R_{d_{L^{p / L}} L_{2}} \psi_{I_{2}}(p) \\
& -S_{d_{L} p / L_{1}} \psi_{I_{3}}(p)
\end{aligned}
$$

where

$$
\begin{aligned}
& I_{1}=\left\{p_{1}, p_{2} \mid \frac{L_{2} p_{1}+L_{1} p_{2}}{d_{L}}=p \bmod N\right\} \\
& I_{2}=\left\{p \mid p=0 \bmod L_{2} / d_{L}\right\} \\
& I_{3}=\left\{p \mid p=0 \bmod L_{1} / d_{L}\right\} .
\end{aligned}
$$

The first sum in (B.1) can be simplified considerably by a better characterization of the index set $I_{1}$. Specifically, it is shown in Appendix C that $I_{1}$ of (B.2) is given equivalently by (3.15) and (3.16) where $0 \leqslant k \leqslant K_{2} N_{1} d_{L} / L_{1}-1$. Thus, the double summation over $p_{1}$ and $p_{2}$ becomes a single summation over $k$. The result is a reduction by a factor of $L_{1} L_{2} / d_{L}{ }^{2}$ in the computation required to evaluate the summation (this factor is 193 for the numerical example of Section 4.0).

\section{APPENDIX C}

If we rewrite the specification of $I_{1}$ in (B.2) as

$$
\frac{L_{2} p_{1}+L_{1} p_{2}}{d_{L}}=p+n N
$$

for some $n$ and fix $n$, we recognize (C.1) as a first degree Diophantine equation [10, p. 96]. All solutions for $p_{1}$ are of the form of (3.15) where $p_{1} *$ is any particular solution. To verify that the unique $p_{1}$ * satisfying (3.17) is a solution to (C.1) for any $n$, note that since $L_{1} / d_{L}$ divides $N$

$$
\begin{aligned}
L_{2} p_{1} * / d_{L} & =p \bmod L_{1} / d_{L} \\
& =(p+n N) \bmod L_{1} / d_{L} \\
& =p+n N+d L_{1} / d_{L}
\end{aligned}
$$

which is of the form of (C.1) with $p_{2}=-d$. The fact that $p_{1}$ * is unique follows from the fact that $L_{1} / d_{L}$ and $L_{2} / d_{L}$ are relatively prime. To establish the range of $k$ in (3.15), observe that since

$$
0 \leqslant p_{1} * \leqslant L_{1} / d_{L}-1
$$

and since in (B.1) the range of $p_{1}$ becomes, substituting from (3.15),

$$
0 \leqslant p_{1} *+L_{1} k / d_{L} \leqslant K_{2} N_{1}-1
$$

or

$$
-1<-\frac{p_{1}{ }^{*} d_{L}}{L_{1}} \leqslant k \leqslant \frac{K_{2} N_{1} d_{L}}{L_{1}}-\frac{\left[p_{1}^{*}+1\right] d_{L}}{L_{1}} .
$$

Since

$$
0<\frac{\left(p_{1}^{*}+1\right) d_{L}}{L_{1}} \leqslant 1
$$

it follows that

$$
0 \leqslant k \leqslant \frac{K_{2} N_{1} d_{L}}{L_{1}}-1
$$

Finally, when (3.15) is substituted into (C.1) we can solve for $p_{2}$,

$$
p_{2}=\frac{d_{L} p-L_{2} p_{1}^{*}}{L_{1}}-\frac{L_{2}}{d_{L}} k+n K_{1} N_{2}
$$

which in view of the range of $p_{2}$ in (B.1) is satisfied for precisely one $n$, that given by (3.16) and (3.18).

\subsection{ACKNOWLEDGMENT}

The author gratefully acknowledges several useful discussions with D. L. Duttweiler on this problem.

\section{REFERENCES}

1. J. Tierney, C. M. Rader, and B. Gold, "A Digital Frequency Synthesizer," IEEE Trans. Audio and Electroacoustics, Vol. AU-19, p. 48 (1971).

2. Jerzy Gorski-Popiel, Editor, Frequency Synthesis-Techniques and Applications, New York: IEEE Press, 1975.

3. J. Noordanus, "Frequency Synthesizers-A Survey of Techniques," IEEE Trans. Comm. Tech., Vol. COM-17, p. 257, April, 1969.

4. V. F. Kroupa, Frequency Synthesis, New York: Wiley, 1973.

5. V. Manassewitsch, Frequency Synthesizers Theory and Design, New York: Wiley 1976.

6. J. F. Oberst, "Generalized Phase Comparators for Improved PhaseLocked Loop Acquisition," IEEE Trans. Comm., Vol. COM-19, December, 1971 , p. 1142.

7. G. C. Gillette, "The Digiphase Synthesizer," Frequency Technology, p. 25, August 1969

8. A. J. Lincoln, S. Even, and M. Cohn, "Smooth Pulse Sequences," Proc. Third Annual Princeton Conference on Information Sciences and Systems, 1969.

9. C. J. May, U.S.P. 4, 034, 302, July 5, 1977.

10. B. M. Stewart, Theory of Numbers, New York: Macmillan, 1964.

11. D. L. Duttweiler and D. G. Messerschmitt, "Analysis of Digitally Generated Sinusoids with Application to A/D and D/A Converter Testing," IEEE Trans. Comm., Vol. COM-26, pp. 669-675, May 1978.

David G. Messerschmitt (S'65-M'68), for a photograph and biography, see page 675 of the May 1978 issue of this TRANSACTIONS. 